\title{
Three-dimensional retinal image stability during visual tilt discrimination in the rabbit
}

\author{
J. Van der Steen, J.H. Reuter and M.W. Van Hof \\ Department of Physiology I, Faculty of Medicine, Erasmus Universiteit, Rotterdam (The Netherlands) \\ (Received 27 February 1990) \\ (Accepted 15 May 1990)
}

Key words: Visual discrimination; Eye movement; Retinal image stability; Rabbit

\begin{abstract}
Rabbits trained to discriminate vertical vs. oblique striations are unable to discriminate angular differences of $5^{\circ}$. In the present study the instabilities around the roll axis of the eyes were measured during visual discrimination. The results indicate that these instabilities are one of the causes of the rabbit's inability to discriminate angular differences of $5^{\circ}$.
\end{abstract}

\section{INTRODUCTION}

Rabbits are able to discriminate striated patterns of different orientation ${ }^{10}$. In several studies in which rabbits were trained on a tilt discrimination, it was found that performance declined to chance level when the angular separation from vertical was 5 degrees ${ }^{11,13}$.

Behavioural experiments have shown that rabbits, during visual discrimination, fixate the patterns with the temporal retina ${ }^{4,12}$ of their laterally sited eyes. This means that any instability around the fronto-occipital (roll) axis of the skull will influence the orientation of the images projected onto the retina. The question arises whether the inability to discriminate striated patterns with angular differences of 5 degrees or less is likely to be due to such instabilities.

In a previous study in freely moving rabbits it was found that positional instabilities of the eyes around the roll axis of $+5^{\circ}$ and $-5^{\circ}$ occur frequently ${ }^{8}$. To date, however, there has been no exact determination of the degree of ocular stability in rabbits during tilt discrimination.

In the present study rabbits were tested over a range of varying angular tilt discriminations. At the same time horizontal, vertical and torsional head and eye movements were recorded by means of the search coil technique ${ }^{1,5,8}$.

\section{MATERIALS AND METHODS}

Five Dutch-belted rabbits were trained to discriminate striated patterns of different orientation. The pattern discrimination box was essentially the same as described previously ${ }^{10}$. The animals had to choose between 2 patterns, one of which was rewarded, a sample of which is shown in Fig. 1. Both patterns could be adjusted to any tilt. Two guillotine doors were positioned in the box in front of the 2 choice gates. One of the doors was made of clear plexiglass, the other out of

Correspondence: J. Van der Steen, Department of Physiology I, Faculty of Medicine, Erasmus Universiteit, P.O. Box 1738, 3000 DR Rotterdam, The Netherlands. 


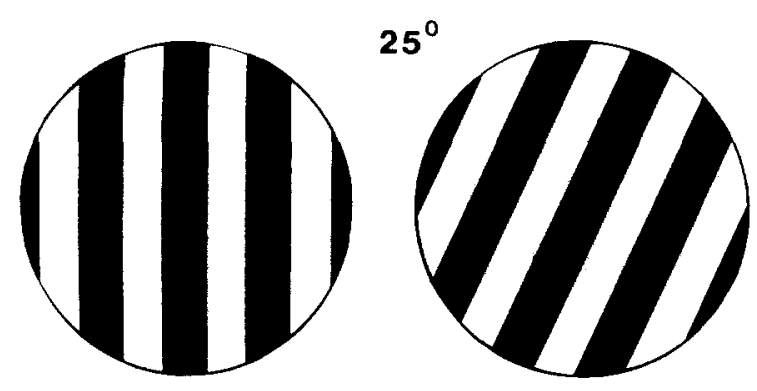

Fig. 1. Striated patterns as seen by the animal. Diameter $10 \mathrm{~cm}$, width of the striations $1.25 \mathrm{~cm}$. In all cases the vertical striations were rewarded. Angular differences during the actual experiments was $45^{\circ}, 25^{\circ}, 15^{\circ}$, or $10^{\circ}$. The vertical striations were projected randomly left or right. The oblique striations were randomly clockwise or counterclockwise rotated.

opaque perspex. By first opening the opaque door the animal could see both patterns, but not access the gates. When the plexiglass door was raised, the gate displaying the rewarded pattern could be accessed by the rabbit. Thus the raising of the opaque door defined the onset of visual stimulation, and the subsequent raising of the transparent door defined the opportunity to execute a choice. The temporal sequence of the lifting of each guillotine door and the animal's response to the choice gates were recorded by means of electronic switches.

Initially the animals were trained to discriminate vertical vs. horizontal striations (vertical rewarded). Responding to the panel with the nonrewarded pattern terminated the trial, as a noncorrection procedure was used. In all cases 40 trials were given per day. After the $90 \%$ correct level had been reached, training was continued with vertical vs. oblique striations (randomly clockwise or counter clockwise). Equal numbers of presentations of vertical vs. $45^{\circ}, 25^{\circ}, 15^{\circ}$ and $10^{\circ}$ striations were given in random order. At this point scleral search coils were attached to the animals' eyes ${ }^{1,8}$. After the operation the animals were retrained to their preoperative criterion level.

Horizontal, vertical and torsional head and eye movements were recorded by means of the technique of search coils in an A.C. magnetic field. This combines the phase angle detection technique for measuring horizontal head and eye movements ${ }^{1}$, with the amplitude detection tech- nique for measuring roll and pitch rotations of the head and eye ${ }^{5}$.

In short, a vertically and a horizontally wound coil were attached to one of the eyes of each rabbit. A similar set of coils was attached to the cable with the head connectors. A homogeneous magnetic field was used to allow precise recording of the horizontal angular head and eye position over a range of $360^{\circ}$, with the vertical coils using the phase angle detection technique. The horizontally oriented head and eye coils were connected to a pair of dual phase lock-in amplifiers, to measure the roll and pitch angular position of the eye and head in space (Fig. 2).

The signal from the vertically oriented head coil was used as the phase reference input to both amplifiers, in order to let the axes of the roll and pitch movements rotate with the horizontal head movements. A full description of this method has been given before ${ }^{8}$.

The behavioural experiments and the eye movement recordings were carried out in a dimly illuminated room. The hand-controlled wooden discrimination box was located inside the magnetic field. The box was provided with electronic switches to indicate the moments in time at which the various doors were opened. The states of these switches were converted into an analog voltage level. This and the eye and head position

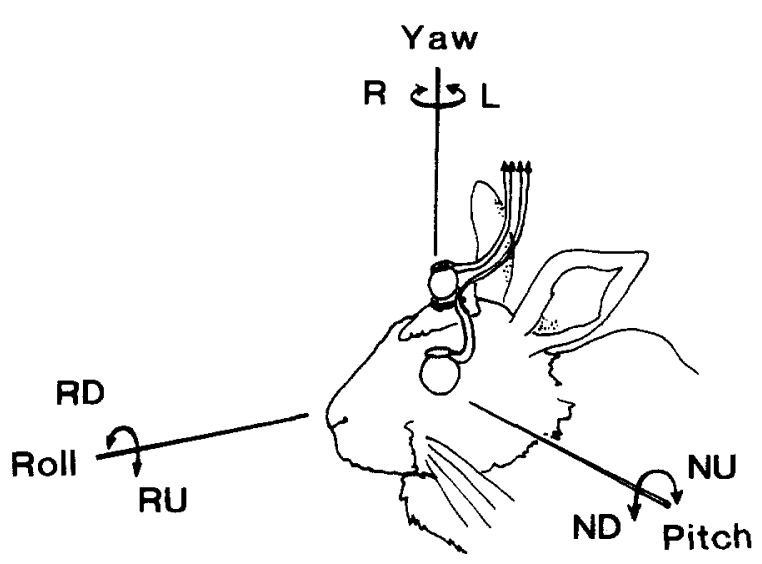

Fig. 2. A vertically and a horizontally wounded coil was implanted on one eye of each rabbit. A similar set of coils was attached to the skull. The coils were connected to a recording system by means of a cable which did not interfere with the movements of the animals. 
signals were sampled on-line at a rate of 125 samples/s on a PDP 11-73 computer. In addition all signals were charted on a penrecorder (Grass polygraph).

\section{RESULTS}

At first the animals were trained to discriminate vertical vs. horizontal striations. After the $90 \%$ correct level had been reached training was continued with vertical vs. oblique striations. In all cases 40 trials were given per day. Then the search coils were implanted. After the operation they were retrained with vertical vs. horizontal striations to their preoperative criterion level. After this, equal numbers of presentations of vertical vs. $45^{\circ}, 25^{\circ}, 15^{\circ}$ and $10^{\circ}$ striations were given in random order on 2 consecutive days.
This means that in the 5 rabbits studied a total of 400 trials were given. In 376 out of these 400 trials the correct choice was made.

Watching the animals while they were making a correct choice, it was seen that during the intertrial interval, i.e. the period with both screens lowered, the animals were usually sitting with the head near the place where either the left or the right pattern was going to appear.

In most cases, the fronto-occipital axis was approximately perpendicular to the screens. When the opaque screen was lifted and the rewarded pattern appeared in front of the animal, it did not make any other orienting movements until the transparent screen was lifted and the gate with the food reward could be accessed. If, on the other hand, the unrewarded pattern appeared in front of the animal, it reoriented itself by making
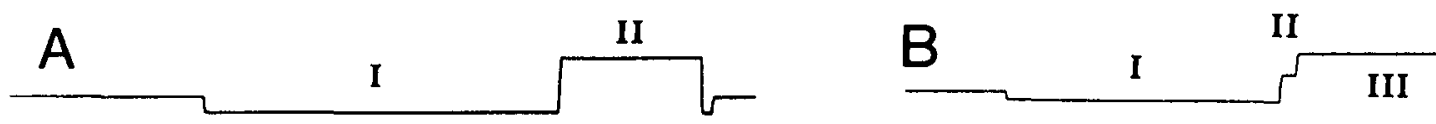

III
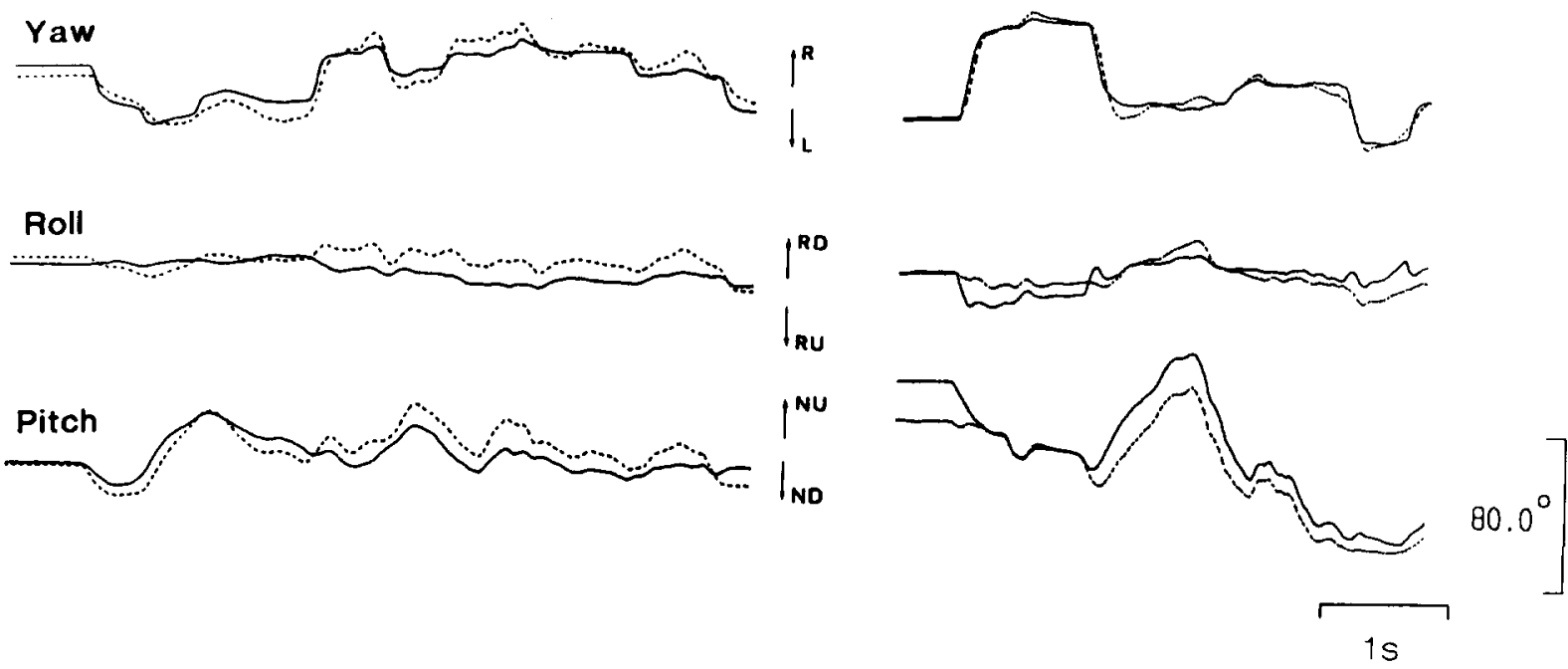

Fig. 3. Two examples of digitized recordings of single trials. The upper trace indicates the position of the sliding doors and the gates carrying the striated patterns. During phase I the non-transparent door was lifted so that the animals could see the patterns. After that the clear plexiglass door was lifted (phase II). During phase III the animal opened the gate which carried the vertical striations. The other 3 traces show the head (dashed lines) and eye (solid lines) positions for yaw, roll and pitch resp.: $\mathrm{R}=$ right, $\mathrm{L}=$ left, $\mathrm{RD}=$ right side down, $\mathrm{RU}=$ right side up, $\mathrm{NU}=$ nose up, $\mathrm{ND}=$ nose down. $\mathrm{A}$ is an example of a trial in which the rewarded pattern appeared in front of the animal. In B the unrewarded pattern appeared in front of the animals. As 'the yaw' trace shows, the animal moved to the other side. In the upper trace of B the deflection which indicates phase I begins at the moment when the opaque door is fully lifted. As seen in the graph which indicates the horizontal movements (second trace) the reorienting movement was started already when part of the target was visible. 
a single horizontal head movement towards the other pattern already before the transparent screen was opened.

Figure 3 gives 2 typical examples of the threedimensional eye and head movements during the visual discrimination task. The left panel shows an example of an animal which during a discrimination trial had oriented itself towards the right pattern $300 \mathrm{~ms}$ before the opaque screen was raised (upper trace, marked phase I). Since the rewarded pattern appeared in front of the animal, it did not make any substantial reorienting horizontal head and eye movements, until the transparent screen (phase II) had been raised and the animal had opened the gate (phase III).

The right panel of Fig. 3 shows an example of a trial at which the animal was also oriented to the right pattern before opening of the opaque door. Now the unrewarded pattern appeared in front of the animal, and a single horizontal reorienting head and eye movement was made.

Under the circumstances shown in the second example of Fig. 3 the time between the raising of the opaque screen and the animals's reorienting horizontal head movement was always less than $500 \mathrm{~ms}$. Thus it is likely that the decision concerning which gate to be opened was made during this time period. Alternatively, at those trials in which the rewarded pattern appeared in front of the animal, there was no reaction until the transparent screen was elevated. However, it is a

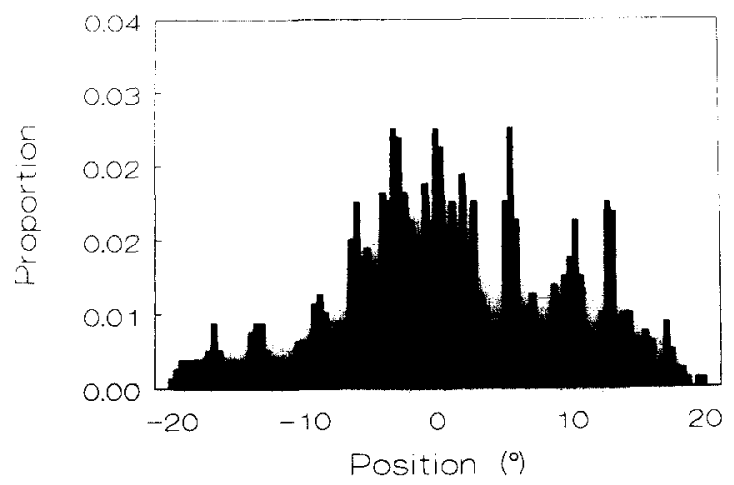

Fig. 4. Distribution of all roll positions measured in 5 animals during the periods of correct decision making in 40 trials. In random sequence each angular difference $\left(45^{\circ}\right.$, $25^{\circ}, 15^{\circ}$ and $10^{\circ}$ ) was presented 10 times. Individual S.D.s were $5.25^{\circ}, 7.17^{\circ}, 7.45^{\circ}, 8.07^{\circ}$ and $10.8^{\circ}$. reasonable assumption that also at these trials the decision making did not take longer than $500 \mathrm{~ms}$ after the patterns became visible.

From this point of view we assumed that the crucial information used for choice decisionmaking derives from the initial $500 \mathrm{~ms}$ period after presentation of the visual displays or shorter if a head movement to the other side was made earlier. If this were the case then retinal stability during this period would be an important factor to analyse as a possible determinant of the accuracy of tilt discrimination.

In Fig. 4 the distribution of all roll positions during the periods of correct decision making are shown. The postion of the eye was sampled every $8 \mathrm{~ms}$ during the first $500 \mathrm{~ms}$ after the patterns became visible. If was found that the instability did not differ significantly with angular differences of $45^{\circ}, 25^{\circ}, 15^{\circ}$ and $10^{\circ}$. Therefore the roll position measurements of all correct trials of all 5 rabbits were averaged and found to be $-1.5^{\circ}$. The standard deviation of all position measurements was $\pm 7.60^{\circ}$.

\section{DISCUSSION}

In an earlier study it was shown that the occipital cortex in the rabbit plays a crucial role in visual discrimination of striated patterns ${ }^{14}$. Under the test conditions described above, the striated patterns project onto the temporal part of the laterally sited eyes of the rabbit ${ }^{4,10}$. This means that instabilities around the frontooccipital axis of the eye - 'roll' - will interfere with the proper alignment of the retinal projection within the coordinate system of the occipitocortical map ${ }^{7}$. The larger the instability around the fronto-occipital axis of the eye, the larger the discrepancy between orientation in space and in the cortical map. As mentioned before, the position of the eye was sampled every $8 \mathrm{~ms}$ during the first $500 \mathrm{~ms}$ after the patterns became visible. It was found that the standard deviation of these position measurements with respect to vertical was $7.60^{\circ}$. As a consequence, when rabbits are asked to discriminate between vertical and $5^{\circ}$ oblique striations, in about half of these measurements $(z=0.66)$ the spatial and cortical coordi- 
nates will differ more than the angular difference between the 2 striated patterns. This instability around the fronto-occipital axis could be the cause of the rabbit's inability to discriminate angular differences of $5^{\circ}$, provided that one assumes that the rabbit is not capable of integrating visual information provided by the retinal image and positional information provided by the vestibular system. If the rabbit were able to integrate visual and vestibular inputs for the decision making it might be possible to detect angular differences with greater precision than the instability suggests. From the above described results it follows that the rabbit does not use any systematic visual scanning of the targets. This is in accordance with a previous study in which it was found that rabbits are able to discriminate between striated patterns of different orientation which were illuminated by means of an electronic stroboscope ${ }^{15}$.

On the other hand, Swadlow has described that the tuning range of orientation sensitive units in the binocular visual cortex of the rabbit is minimally $20^{\circ}$. This suggests a low resolution with respect to tilt detection at a cortical level. Therefore the instability is not necessarily the only factor which limits the rabbit's ability to discriminate striations of different orientation. These results obtained in the rabbit are in marked contrast with those obtained in human subjects. It has been shown that a sitting person, in the absence of visual context, can set the subjective vertical with a precision of about $1^{\circ}-2^{\circ 3}$. As in the rabbit, the SD of the subjective vertical appears to be of a magnitude similar to that of the long-term fluctuations of ocular torsion ${ }^{2}$.

\section{REFERENCES}

1 Collewijn, H., Eye- and head movements in freely moving rabbits, J. Physiol. (Lond.), 266 (1977) 471-498.
2 Collewijn, H., Van der Steen, J., Ferman, L. and Jansen, T.C., Human ocular counterroll: assessment of static and dynamic properties from electromagnetic scleral coil recordings. Exp. Brain Res., 59 (1985) 185-196.

3 Collewijn, H., Ferman, L. and Van den Berg, A.V., The behavior of human gaze in three dimensions. In: Representation of three-dimensional space in the vestibular oculomotor and visual systems, Ann. N.Y. Acad. Sci, 545 (1988) 105-127.

4 Lagers- Van Haselen, G.C. and Van Hof, M.W., The temporal retina and visual discrimination in the rabbit, Behav. Brain Res., 28 (1988) 253-257.

5 Robinson, D.A., A method of measuring eye movements using a scleral search coil in a magnetic field, IEEE Trans. Biomed. Electron. 10 (1963) 137-145.

6 Swadlow, H.A., Efferent neurons and suspected interneurons in binocular visual cortex of the awake rabbit: receptive fields and binocular properties, J. Neurophysiol., 59 (1988) 1162-1188.

7 Thompsson, J.M., Woolsey, C.N. and Talbot, S.A., Visual areas I and II of the cerebral cortex of the rabbit, J. Neurophysiol., 13 (1950) 277-288.

8 Van der Steen, J. and Collewijn, H., Ocular stability in the horizontal, frontal and sagittal planes in the rabbit, Exp. Brain Res., 56 (1984) 263-274.

9 Van der Steen, J. and Van Hof, M.W., The relation between the threshold of tilt discrimination and retinal image stability in the rabbit, J. Physiol. (Lond), 420 (1990) $107 \mathrm{P}$.

10 Van Hof, M.W., Discrimination between striated patterns of different orientation in the rabbit, Vision Res., 6 (1966) 89-94.

11 Van Hof, M.W., The accuracy of tilt discrimination in the rabbit, Behav. Brain Res., 4 (1982) 315-318.

12 Van Hof, M.W. and Lagers-Van Haselen, G.C., The retinal fixation area in the rabbit, Exp. Neurol., 41 (1973) 218-221.

13 Van Hof, M.W. and Wiersma, C.A.G., The angular threshold of discrimination for striated patterns of different orientation in the rabbit, Vision Res., 7 (1967) 265-270.

14 Van Hof, M.W., Van Hof-Van Duin, J. and Hobbelen J.F., Visual discrimination after bilateral removal of the visual cortex in the rabbit, Behav. Brain Res., 9 (1983) 257-262.

15 Van Hof, M.W., De Vos-Korthals, W.H. and Lagers-Van Haselen, G.C., Discrimination of flicker gratings of different orientations in the rabbit, Behav. Brain Res., 20 (1986) 152-153 (Abstract). 